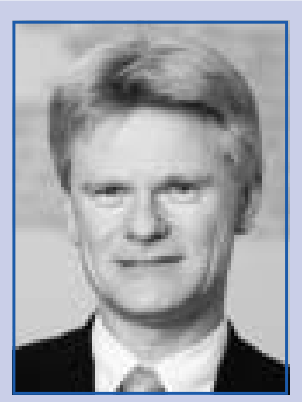

Dipl.-Ing. Dr. techn. Werner Adelberger ist Senior Vice President bei der BMW Group, München, und verantwortlich für den Bereich Controlling Ressort Forschung und Entwicklung und Produktprojekte.

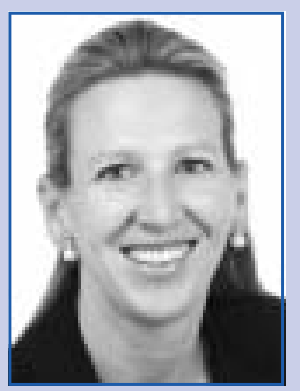

Dr. Nicole Haft-Zboril ist Senior Vice President bei der BMW Group, München, und verantwortlich im Bereich Einkauf und Lieferantennetzwerk für Strategie, Projekteinkauf, Wertstrom- und Komplexitätsmanagement.

\title{
Systematischer Ansatz zur projekthaften Steuerung von Entwicklungskosten
}

\author{
Werner Adelberger und Nicole Haft-Zboril
}

Für die Steuerung von Entwicklungskosten wird ein Ansatz vorgestellt, der es erlaubt, in frühen Phasen mit hinreichender Güte Konzepte einfach zu bewerten. Kern sind die systematische Ermittlung von Kostentreibern auf der Datenbasis abgeschlossener Projekte und eine vereinbarte jährliche Effizienzsteigerung. So wird eine frühzeitige Konvergenz von aus Marktpotenzialen abgeleiteten Aufwandszielen und bewerteten technischen Lösungen effektiv unterstützt.

\section{Ableitung von wettbewerbs- fähigen Zielansprüchen in der Automobilindustrie}

Kostendruck und Wettbewerb in der Automobilindustrie sind hoch. Mit jeder Generation steigen die Anforderungen an vergleichbare Fahrzeuge deutlich. Die Gründe hierfür sind erhöhte gesetzliche Anforderungen bei Crash-Tests, Emissionen, Fußgängerschutz sowie steigende Kundenerwartungen im Bereich von Infotainment, Komfort und Wertigkeit. Wegen des intensiven Wettbewerbs lassen sich Preiserhöhungen für Nachfolgeprojekte am Markt kaum durchsetzen (vgl. Hab/Wagner, 2013, S. 9). Daraus entsteht Kostendruck in allen Bereichen. Dies gilt auch für die Aufwendungen der Entwicklung, sodass der Anspruch besteht, durch Effizienzsteigerungen funktionale Mehrungen quasi ohne Mehrkosten gegenüber dem Vorgänger zu realisieren. Bei $B M W$ wurde ein System entwickelt, mit dem Ziele für Entwicklungskosten zukünftiger Fahrzeugprojekte so abgeleitet werden, dass diese Effizienzsteigerung klar vorgegeben und nachgehalten werden kann. Ziel dieses Artikels ist es, Logik, Ausgestaltung, Anwendung und Grenzen dieses Steuerungsansatzes vorzustellen.

Den Entwicklungskosten kommt in der Automobilindustrie eine besondere Bedeutung zu. Es stellt sich hier die Aufgabe einer analytisch nachvollziehbaren Ableitung von anspruchsvollen, aber realistischen Zielwerten in besonderem Maße, denn früh im Projektverlauf werden wesentliche Produkteigenschaften und damit auch Aufwendungen festgelegt. Ein- mal getroffene Entscheidungen sind nur sehr schwer korrigierbar und führen bei Fehlentscheidungen $u$. U. zu hohen sunk cost. Ehrlenspiel spricht in diesem $\mathrm{Zu}-$ sammenhang vom „Paradoxon der Konstruktion“: In den frühen Phasen der Produktentstehung können die Produkteigenschaften mit geringem Aufwand geändert werden, gleichzeitig sind ihre Auswirkungen $\mathrm{zu}$ diesem Zeitpunkt am schwierigsten bewertbar (vgl. Ehrlenspiel, 2009, S. 193). Klare Prämissen und realistische handlungsleitende Zielwerte sind daher eine wichtige Voraussetzung für tragfähige Projektbewertungen (vgl. Schorb, 1994, S. 79 f.) sowohl in frühen Phasen strategischer Weichenstellung wie auch in den folgenden Realisierungsphasen.

Daher ist es essenziell, für die Ableitung der Entwicklungskostenziele eine Systematik zu finden, die eine möglichst universelle Einsetzbarkeit, hinreichende Genauigkeit und die nachhaltige Verankerung von Best Practice-Ansätzen bei vertretbarem Aufwand sicherstellt. Gleichzeitig sind spezifische Rahmenbedingungen und Anforderungen an die Entwicklung eines Fahrzeugs einzubeziehen. „Best Practice“ bezeichnet in diesem $\mathrm{Zu}$ sammenhang die effizientesten und effektivsten Entwicklungsprozesse von Fahrzeugprojekten im hausinternen Projektvergleich.

Solch ein System wird bei BMW als „Führungssystem “ bezeichnet. Führungssysteme begleiten und leiten die Produktentstehung und stellen sicher, dass vom Markt diktierte Preis- und damit Kostenniveaus mit den aufwandstreibenden 
technischen Prämissen des Projekts in Einklang gebracht werden. Im Verlauf der Produktentstehung wird dafür gesorgt, dass die geforderten Zielwerte realistisch erreichbar sind, dem technischen Konzept entsprechen und die geforderte Projektrentabilität mit den vereinbarten Entwicklungskosten erreicht werden kann. Im Zuge dessen werden auch Projekte rechtzeitig erkannt, die betriebswirtschaftlich nicht zielführend sind.

\section{Best Practice als Ausgangs- punkt für ein zielkostenorien- tiertes Führungssystem}

Für die systematische Ableitung von Zielen für Projektkosten bieten sich unterschiedliche Methoden an, die im Folgenden kurz erläutert werden sollen.

In jedem Unternehmen gibt es ausreichend im Ist erfasste Daten historischer Projekte inkl. der genauen Prämissen sowie punktuelle Wettbewerbsvergleiche aus Benchmarkanalysen. Experten, sprich erfahrene Fachbereichsvertreter aus der Entwicklung, aber auch aus dem Produktcontrolling, haben ein „gutes Gefühl“ für die wesentlichen Kostentreiber bzw. Stellhebel zur Kostenreduzierung in einem Projekt. Es ist naheliegend, dieses Wissen systematisch $\mathrm{zu}$ erfassen und in einem Führungssystem standardisiert zu hinterlegen. Wesentliche Voraussetzung für das Erarbeiten handlungsleitender Zielwerte ist hierbei ein genaues Verständnis des Aufwands, der für die Umsetzung der gegebenen Prämissen notwendig ist. Die Prämissen eines Fahrzeugprojekts beschreiben den Entwicklungskontext wie die Entwicklung eines eigenständigen Projekts, eines Derivats auf einer bestehenden Architektur oder den Anteil an baukasten- bzw. derivatspezifischen differenzierenden Umfängen. Prämissen definieren aber auch Faktoren wie den Eigenleistungsanteil der Entwicklung oder die geforderte Variantenvielfalt zur Erfüllung entsprechender Marktanforderungen. Ein Effizienzanspruch lässt sich erst dann konsistent durchsetzen, wenn dieser Aufwand für die Projektdurchführung mit phasenadäquater Genauigkeit bekannt ist.

In der Literatur lässt sich eine Vielzahl von Methoden zur Aufwandsschätzung finden. Ihr Fokus liegt zumeist bei der Abschätzung von Material- und Herstellkosten oder aber beim Erstellungsauf- wand in der Softwareentwicklung. Ihnen allen gemein ist das grundsätzliche Spannungsfeld, möglichst „harte“, d. h. verlässliche Werte zu generieren - wie sie die Ableitung von einer Ist-Datenbasis ermöglicht - gleichzeitig aber auch für die jeweiligen Prämissen maßgeschneiderte Werte abzugeben, wie sie sich bspw. aus einer Bottom-up-Planung oder Expertenschätzung ergeben. Nicht zuletzt ist der benötigte Zeiteinsatz bei der Abschätzung ein bestimmender Faktor, denn „Entwickler und Konstrukteure müssen auch zur eigenen Arbeit kommen" (Ehrlenspiel, 2009, S. 210).

In den vergangenen Jahrzehnten haben sich neben klassischen Expertenschätzungen und Bottom-up-Planungen drei grundsätzliche Klassen von Aufwandsschätzmethoden herauskristallisiert (vgl. Burghardt, 2005, S. 156 ff.; Schlechtweg, 2009, S. 29 ff.). Erstens Vergleichsmethoden, die in der Planung befindliche Projekte mit in der Vergangenheit ausgeführten Projekten vergleichen, mit dem Ziel, Aussagen über Kosten abzuleiten. Zweitens Kennzahlenmethoden, die meist lineare Zusammenhänge bestimmter Einflussfaktoren zum Aufwand darstellen, wie Stunden per lines of code bei der Softwareentwicklung. Drittens algorithmische Methoden, die anhand statistischer Zusammenhänge Schätzwerte als Ergebnis mathematischer Funktionen liefern. Ihnen ist auch die in diesem Artikel vorgestellte Methodik zuzuordnen. Unter den algorithmischen Methoden ist besonders die Untergruppe der parametrischen Methoden von Bedeutung. Prominente Beispiele sind hier das COCOMO-Verfahren und IBM's FunctionPoint-Methode (vgl. Burghardt, 2006, S. 167 ff.). Beide Verfahren fokussieren auf die Aufwandsschätzung bei der Softwareentwicklung.

In der Automobilindustrie haben sich je nach Art der Steuerungsgrößen - zwei unterschiedliche Ansätze als praktikabel erwiesen: ein parametrischer Ansatz, geeignet für Entwicklungs-, Einkaufs- und Planungskosten sowie ein Stellhebel-orientierter Ansatz für Materialkosten und Investitionen. Der Stellhebel-orientierte Ansatz stellt eine Kombination aus Vergleichsmethode und Bottom-up-Ausplanung mit standardisierter Prüfung von Kostensenkungspotenzialen dar.

Am Beispiel der Entwicklungskosten werden nun die Grundzüge eines parametrischen Ansatzes vorgestellt.

\section{Standardisierung der Zielab- leitung für Entwicklungskosten über die Modellierung relevanter Einflussparameter}

Jedes Entwicklungsprojekt für Fahrzeuge kann grundsätzlich in Modulen dargestellt werden, die einzelne Komponenten und Funktionsumfänge (z. B. Sitze, Cockpit, Motor) bzw. spezifische Abläufe im Entwicklungsprozess (z. B. Erprobung Gesamtfahrzeug) abbilden. Im konkreten Fall werden je bewertetem Projekt 60 derartiger Module definiert, mit Zielen belegt, geplant und auch im Ist abgerechnet. Parallel dazu kann nach der Art der Aufwände unterschieden werden: Entwicklungspersonalkapazität, Prototypen und Versuchsfahrzeuge zur Erprobung und Absicherung sowie Entwicklungskosten bei Systemlieferanten, die oft auch im Teilepreis in Form einer Umlage enthalten sind und daher schwer isoliert abgegriffen werden können.

Die Erstellung des Systems folgt für alle Module und alle Aufwandsarten einem stringenten fünfstufigen Prozess: Datenerhebung - Parametrisierung - Modellierung der Standardkostenkurve - Validierung - Anwendung (vgl. Abb. 1). Dies erfolgt in einem Team aus Vertretern der Fahrzeugprojektorganisation, der Entwicklungsfachbereiche, des Controllings und je nach Themenstellung auch des Einkaufs.

Bei der Datenerhebung werden die Daten realisierter Projekte gesammelt und die erfassten Kostendaten auf einen Prämissenstand normiert (z. B. Berücksichtigung der Inflation, Währung, Organisationsstände, Eigenleistungstiefe). Für die nächsten Schritte werden entsprechend repräsentative Projekte für die Ausprägung der Kostentreiber sowie für die Ermittlung der Best Practice selektiert. In diesem Schritt entstehen eine Dokumentation der technischen Inhalte der ausgewählten Projekte sowie eine erste Liste der jeweils relevanten Kostentreiber. Um eine breite statistische Basis zu bekommen, sollten Projekte mit Kostentreibern möglichst unterschiedlicher Ausprägung gewählt werden.

In der folgenden Parametrisierung (vgl. Abb. 2) werden die Einflussgrößen mit den größten Stellhebeln identifiziert und die Wirkung dieser Einflussgrößen auf die einzelnen Aufwandsarten durch Ana- 
lysen und Expertenschätzungen bestimmt. Dabei sollten Einflussgrößen mit einer Hebelwirkung $<5 \%$ nicht betrachtet werden. Am Ende der Parametrisierung hat man stimmige Kostendaten der ausgewählten Projekte und die dazugehörenden Faktorausprägungen (= Kostenfaktoren) aus der Multiplikation der definierten Gewichtungsfaktoren der Kostentreiber des spezifischen Projektes.

Die Liste potenzieller Parameter ist über die Module hinweg abzustimmen. Es er- gibt sich eine Reihe von Kostentreibern, die in fast allen Modulen wirken, wenn auch mit unterschiedlichen Hebeln. Beispiele für modulübergreifende Einflussfaktoren sind: Fahrzeugklasse, Antriebsvarianten (Allrad-, Front-, Heckantrieb), Leadprojekt einer Produktfamilie oder Derivat. Beispiele für modulspezifische Einflussfaktoren sind: Karosserievariante, Grad der Konzeptkommunalität, Anzahl Hinter-, Vorderachsvarianten, Grad virtueller Absicherung.

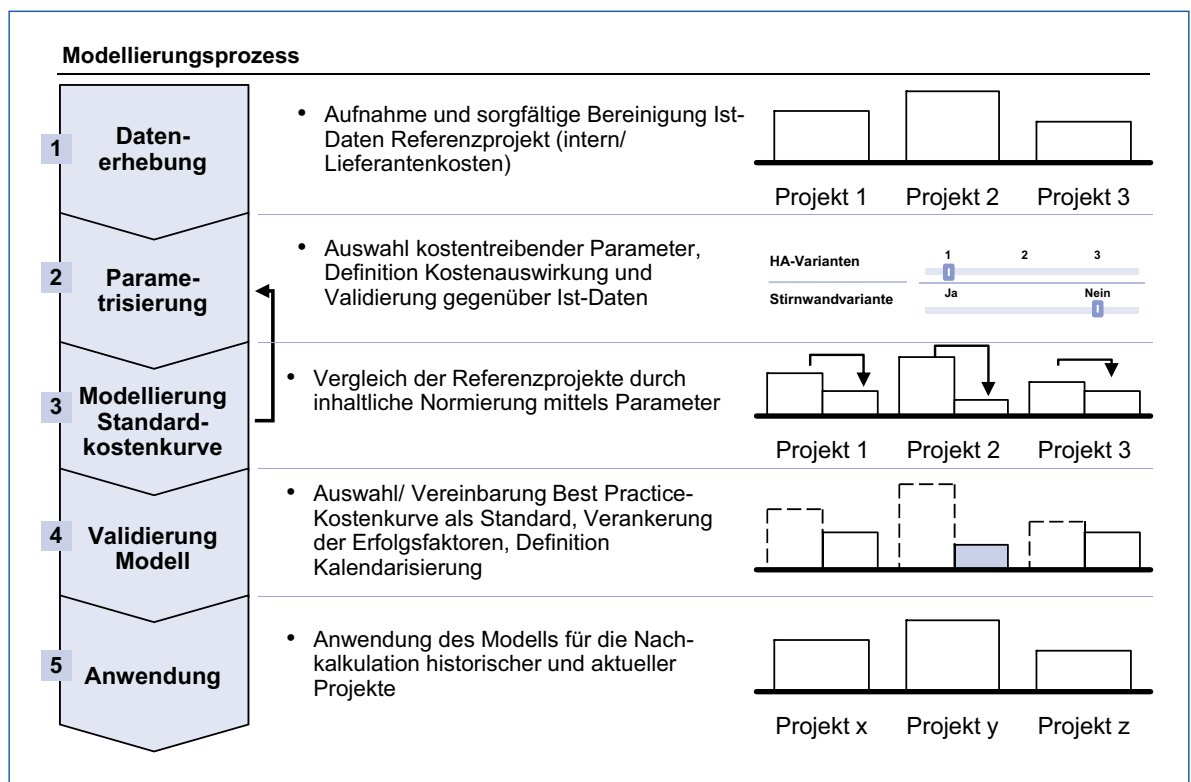

Abb. 1: Parameterbasierte Zielkostenermittlung anhand eines standardisierten Prozesses
Der nächste Prozessschritt ist die Modellierung. Hier werden zunächst alle ausgewählten Projekte auf ein „Standardprojekt" normiert, das im Weiteren die Basis bildet und die Faktorausprägung/den Kostenfaktor 1,0 bekommt. Somit ergibt die Division der stimmigen Kostendaten durch den projektspezifischen Kostenfaktor einen normierten Wert, der die Kosten so darstellt, als wäre die Ausprägung des konkreten Projekts identisch zu dem des Standardprojekts. Das Projekt mit dem niedrigsten Wert war somit am effizientesten, wird zum Referenzprojekt und stellt die Best Practice der ausgewählten Projekte dar. Die Bestimmung der Parameter mit ihren Hebeln auf den Aufwand und die Normierung stellt einen iterativen Prozess dar.

Im nächsten Schritt gilt es die Güte der Modellierung (normiertes Best PracticeProjekt sowie Hebel und Ausprägung der Einflussgrößen) im Rahmen der Validierung an einer „Kontrollgruppe“ aus aktuellen und anderen abgeschlossenen Projekten zu überprüfen. Diese Kontrollgruppe sollte sich von den Projekten, die zur Ableitung der Best Practice herangezogen wurden, klar unterscheiden. Idealerweise sollten z. B. Projekte enthalten sein, die von externen Entwicklungspartnern bereits realisiert wurden oder zur Umsetzung angeboten sind. Dabei kommt es mitunter vor, dass ein Projekt der Kontrollgruppe niedrigere normierte

\section{Durch Normierung der Projekte werden die Effizienzunterschiede erkennbar:}

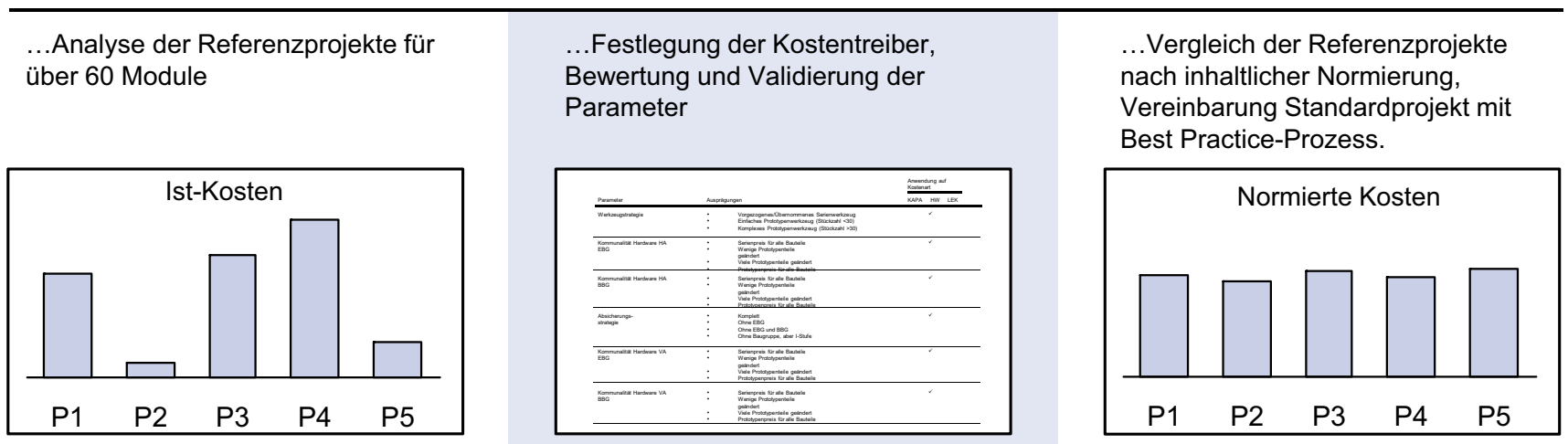

Output:

- Referenzprojekte

- Projektdokumentation
- Kostentreiber pro Modul

- Effizienzmaßnahmen in den Projekten

- Bewertete Kostenparameter
- Festlegung Best Practice

- Erfolgsfaktoren zur Verankerung

- Kalendarisiertes Standardprojekt

- Parametermodell auf Modulebene 


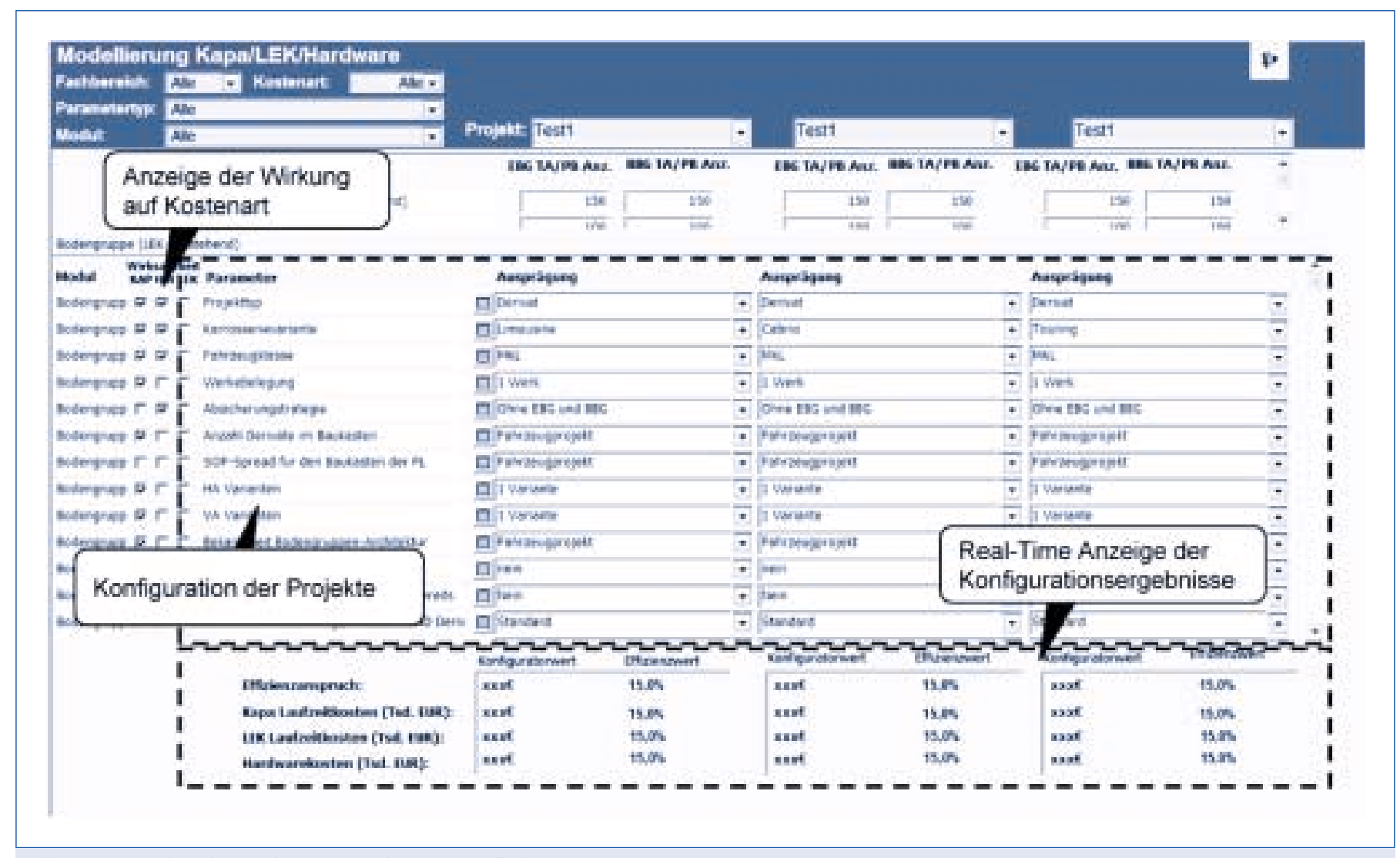

Abb. 3: Screenshot des Tools zur Ermittlung von Kalkulationswerten

Aufwendungen zeigt und damit als Best Practice zu definieren ist. Je nach Ergebnis dieser Analyse wird das Modell entweder freigegeben oder ist erneut zu kalibrieren. Die Erarbeitung und Validierung des Modells erfolgt in funktionsübergreifenden Teams, die Abnahme der Ergebnisse in Qualitätsausschüssen, die mit Führungskräften aller beteiligten Bereiche besetzt sind.

Das Modell wird in einem IT-System (einem sogenannten Entwicklungskostenkonfigurator) hinterlegt und steht dann für die Bewertung neuer Entwicklungsprojekte zur Anwendung bereit. Ein wesentliches Element für die Bewertung neuer Projekte ist ein stimmiges, zentral definiertes Prämissenset für alle Einflussgrößen, das die Anforderungen an das zu bewertende Projekt präzise beschreibt. Die Bewertung erfolgt wieder in interdisziplinären Teams direkt am System (vgl. Abb. 3).

Dieser Prozess liefert im Ergebnis eine inhaltlich fundierte Projektkostenbewertung auf Modulebene, den sogenannten Konfiguratorwert inklusive einer standardisierten Kalendarisierung der Kosten auf die einzelnen Jahre der Entwick- lungszeit. Kernelement der Projektbewertung ist dabei, dass sie den aktuell erreichten Best Practice-Stand in der Projektumsetzung unterstellt. Die erzielbare Genauigkeit eines derartigen Modells liegt bei etwa $90 \%$; d. h., die realen Werte streuen auf Modulebene um ca. +/- $5 \%$ gegenüber dem ermittelten Konfiguratorwert. Für die betriebswirtschaftliche Steuerung eines Fahrzeugprojekts in frühen Entwicklungsphasen, in denen die wesentlichen Entscheidungen getroffen werden, ist dieser Wert ausreichend genau.

\section{Stellhebel der Entwicklungs- effizienz}

Der Konfiguratorwert für ein konkretes Fahrzeugprojekt basiert auf der aktuellen Best Practice, unterstellt aber keine weiteren Effizienzfortschritte für die Zukunft. Um von Fahrzeuggeneration zu Fahrzeuggeneration Freiräume für eine aufwandsneutrale Umsetzung gestiegener Produktanforderungen $\mathrm{zu}$ erreichen, ist ein Effizienzfortschritt von etwa $5 \%$ p. a. erforderlich.
Für die Entwicklungskosten des Nachfolgemodells eines am Markt befindlichen Fahrzeugs ergibt sich damit bei angenommener Funktionsgleichheit und sieben Jahren Modelllaufzeit ein Effizienzanspruch von rund $30 \%$, der sich mit dem für die Automobilindustrie ansetzbaren Effizienzpotenzial deckt (vgl. Bullinger et al., 2003, S. 110).

Es gibt drei große Stellhebel für Effektivitäts- und Effizienzsteigerungen entlang einer Produktentwicklung, die nun näher erläutert werden sollen: Portfolioeffektivität sowie Konzept- und Umsetzungseffizienz (vgl. Abb. 4).

Beim Start jedes Fahrzeugprojektes müssen zunächst die externen Anforderungen in Bezug auf differenzierte Kundenwünsche, allgemeine Marktanforderungen, gesetzliche Regelungen und weitere Rahmenbedingungen klar bestimmt werden (vgl. Hab/Wagner, 2013, S. 62). Im Rahmen des Portfoliomanagements kommt vor allem der Frage nach der Ausschöpfung eines definierten Marktsegmentes mit einem oder mehreren Derivaten, d. h. der Optimierung des Angebotsportfolios, entscheidende Bedeutung zu (vgl. Adelberger/Haft-Zboril, 2013, 


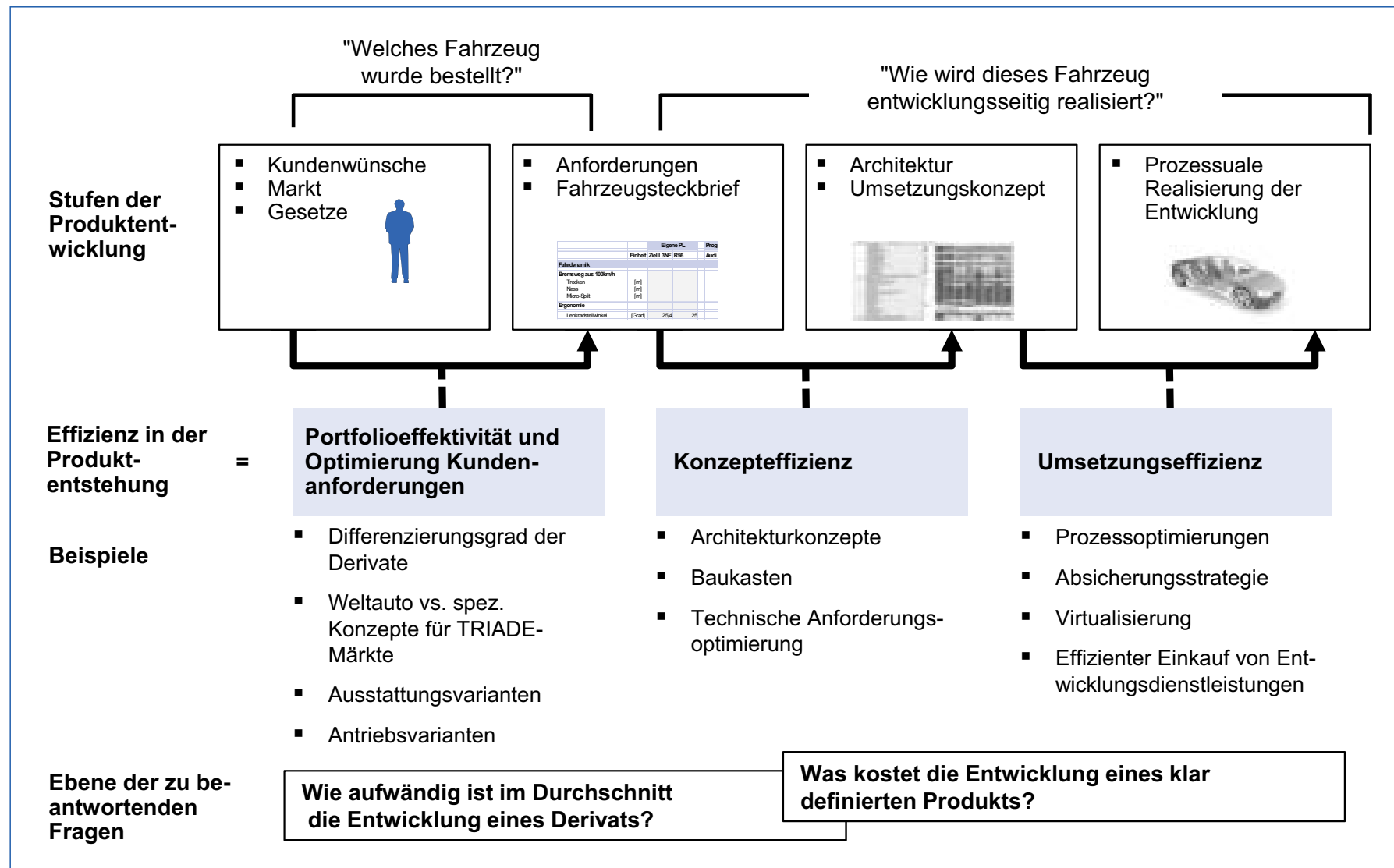

Abb. 4: Stellhebel in der Produktentwicklung

S. $45 \mathrm{ff}$.). Die Portfolioeffektivität zielt auf die optimale Ausschöpfung eines Marktsegments bei optimiertem Ressourceneinsatz. Produkte und deren differenzierende Eigenschaften werden unter der Annahme festgelegt, dass in definierten Segmenten Preise und Herstellkosten in vergleichsweise engen Korridoren festgelegt sind. Damit liegen die erzielbaren Deckungsbeiträge ebenfalls in prognostizierbaren Bandbreiten, was dazu führt, dass der amortisierbare Produktaufwand (Entwicklungskosten, Planungskosten, Investitionen etc.) durch das erzielbare Absatzvolumen gegeben ist.

Die Bandbreite der Entscheidungen reicht von „wollen wir ein 2- oder ein 4türiges Coupé, oder vielleicht doch beide Derivate?“ über „wo wollen wir uns relativ zum Wettbewerb bezüglich Motorleistung, Beschleunigung etc. positionieren?" oder „welches Sonderausstattungsprogramm bieten wir an?" bis zu „welche Wartungsintervalle streben wir an?" oder „wollen wir gewisse Umweltgesetze (z. B. EU 6) bereits vor ihrem eigentlichen Inkrafttreten erfüllen?"
Diese im Rahmen der Portfoliodiskussion definierten Produktanforderungen bestimmen maßgeblich die Aufwendungen. Die Produktbeschreibungen sind dann in technische Anforderungen (im Automobilbereich die sogenannten Lastenhefte) zu übersetzen (vgl. Nagarajah, 2013, S. 319 ff.). Auf diesen basiert die weitere Produktentwicklung mit zwei klar differenzierten Phasen: die Konzeptentwicklung (ca. 4 bis 2,5 Jahre vor Serienstart) und danach die Serienreifeentwicklung (bis zum Start der Serienproduktion) mit jeweils unterschiedlichen Hebeln für deren kostenoptimale Realisierung.

In der Konzeptentwicklung gilt es, entweder eine bestehende Fahrzeugarchitektur für ein neues Derivat zu nutzen oder eine neue Fahrzeugarchitektur zu definieren. Mit einer Fahrzeugarchitektur wird festgelegt, welche Art von Fahrzeugen ( $\mathrm{Li}^{-}$ mousinen, Cabrios, SUV's u. a.) in welchen Dimensionen und Skalierungen (z. B. Radstand, Spurweite, Gewicht, Höhe der Sitzposition, Motorleistung) sinnvoll ausgeleitet werden können. In dieser Phase erfolgt auch die Festlegung zu ver- wendender Modul- oder Komponentenbaukästen. Ziel ist die Konzepteffizienz betriebswirtschaftlich zu optimieren. Die Konzepteffizienz drückt sich im Konfiguratorwert wie folgt aus:

Konzepteffizienz =

\section{Aufwand des neu definierten Derivats} Aufwand eines normierten Standardderivats

Klassenübergreifende Architekturen und volumenstarke Baukastenkomponenten bilden die Voraussetzung, um signifikante Fortschritte in der Konzepteffizienz zu erzielen. So lassen sich z. B. Marktnischen mit geringem Absatzvolumen nur auf $\mathrm{Ba}$ sis einer hohen Konzepteffizienz wirtschaftlich besetzen.

In der darauffolgenden Serienreifeentwicklung geht es nicht nur um das Absichern interner Best Practice, sondern auch um das Erreichen des geforderten Effizienzfortschritts, was in der Messung der Umsetzungseffizienz zum Ausdruck kommt. Wesentliche Stellhebel der Umsetzungseffizienz in Entwicklungsprozessen sind der Einsatz von CAD Systemen, automatisierte Prüfstände, der Verzicht auf Prototypen durch virtuelle Absiche- 
rung von Baugruppen und Fahrzeugen, aber auch der Einkauf von Entwicklungsdienstleistungen in Best Cost-Countries.

Zum Erreichen eines Effizienzanspruchs von $30 \%$ ist die optimale Anwendung aller Effizienzstellhebel notwendig: An die Konzepteffizienz wird ein Anspruch von $15 \%$ gestellt, der sich im Wesentlichen über die Nutzung von Architekturstandards und Baukästen begründet.

Die verbleibenden fünfzehn Prozent sind durch die Umsetzungseffizienz (netto) abzudecken. Über die Umsetzungseffizienz (brutto) ist weiterhin die Inflation auszugleichen, sodass bei einer langfristig angenommenen durchschnittlichen Inflationsrate von 2,5\% p. a. der Anspruch an die Umsetzungseffizienz von einer Fahrzeuggeneration zur nächsten $30 \%$ brutto ist (vgl. Abb. 5).

Die Analyse historischer Projektdaten zeigt, dass auf diese Weise in den letzten fünf Jahren die Entwicklungsaufwände pro Derivat im Durchschnitt sogar etwas über $30 \%$ gesenkt werden konnten.

\section{Berücksichtigung der Umsetzungseffizienz mit dem Anspruch einer regelmäßigen Aktualisierung}

Das Führungssystem für Entwicklungskosten basiert wie dargestellt auf drei Grundelementen: aktuelle Best Practice, Parameter für die wesentlichen Kostentreiber pro Modul und ein klar definierter Anspruch an die Umsetzungseffizienz.

Wie dargestellt erfolgt die Bewertung eines Fahrzeugprojekts durch Anwendung des Entwicklungskostenkonfigurators und besteht aus ca. 60 Modulwerten. $\mathrm{Zu}$ Beginn wird je Modul ein Kostenwert gebildet, der die aktuelle Best Practice eines Referenzprojektes mit einem konkreten Serienanlauftermin widerspiegelt. Dieser Wert wird mit $5 \%$ Effizienzsteigerung p. a. beaufschlagt, um den definierten Anspruch abzubilden. Die Anzahl der anzusetzenden Jahre richtet sich nach dem zeitlichen Abstand des bewerteten Projekts zum Serienanlaufjahr des Referenzprojekts. Der so ermittelte Wert wird Substanzwert genannt, was ausdrückt, dass es sich um einen Best Practice-Wert für die Entwicklung der über die Parameter definierten Produktsubstanz (= Produktinhalt gemäß Produktkonzept) inkl.

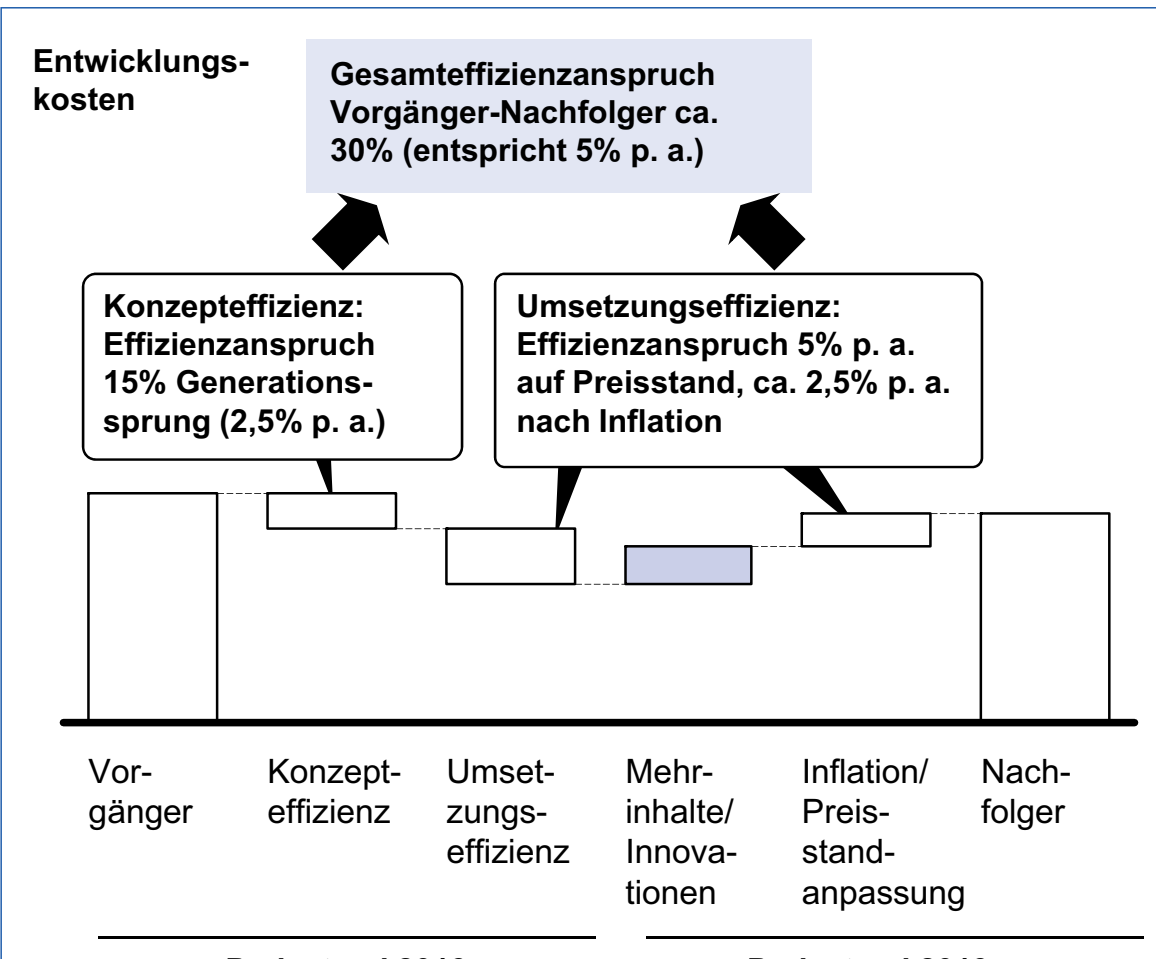

Preisstand 2013

Preisstand $2013+x$ des vereinbarten Effizienzanspruchs handelt. Der Begriff des "Substanzwerts“" wurde bewusst gewählt und löst den Begriff des „Planstands“ ab. Ein Planstand wurde wenig systematisch als Bottomup-Wert der Fachbereiche mit u. U. unterschiedlichen Risikovorhalten ermittelt. Der "Substanzwert" soll eine eindeutige Referenz zur unterstellten Produktsubstanz i. S. der beauftragten Fahrzeugkonfiguration erzeugen. Er ist damit auch vom Begriff des Substanzwerts abzugrenzen, der im Rahmen der betriebswirtschaftlichen Literatur zur Unternehmensbewertung verwendet wird.

Analysen der Vergangenheit zeigen, dass der Effizienzfortschritt nicht linear und auch nicht immer gleichmäßig über alle Entwicklungsbereiche erfolgt. Es ist daher notwendig, jährlich mit den vorliegenden Projektabrechnungen die Best Practice-Werte für alle Module neu zu ermitteln, die dahinter liegenden inhaltlichen Maßnahmen zu dokumentieren und die Datenbasis des Entwicklungskostenkonfigurators entsprechend zu aktualisieren. Nur so kann sichergestellt werden, dass im Sinne eines lernenden Systems die ermittelten Werte der aktuellen Best Practice in den einzelnen Entwicklungsbereichen entsprechen. Dieser Prozess der „Selbstschärfung“ hat unmittel- baren Einfluss auf die Ermittlung von Zielwerten für neue Projekte und muss daher für die Fachstellen sehr transparent und klar nachvollziehbar sein, um die langfristige Akzeptanz dieses Ansatzes im Unternehmen sicherzustellen.

\section{Zusammenfassung}

Das dargestellte Führungssystem hat sich im Unternehmen vor allem durch das Erarbeiten in funktionsübergreifenden Teams schnell etabliert. Es liefert mit vertretbarem Aufwand hinreichend genaue Kalkulationswerte für zukünftige Projekte, die auf allgemein anerkannter Best Practice basieren und verankert nachhaltig den Anspruch auf permanente Effizienzverbesserung in allen Bereichen der Organisation. Es ist vor allem in frühen Phasen ein gutes Werkzeug für die Projektarbeit und schafft eine gemeinsam getragene Basis für ein zielorientiertes, bereichsübergreifendes Vorgehen mit klaren Spielregeln, Rollen und Verantwortlichkeiten von der Produktdefinition bis zur konkreten Projektentscheidung.

$\mathrm{Da}$ es sich um ein statistisches Modell handelt, das auf historischen Daten aufsetzt, hat es allerdings seine Grenzen bei der erstmaligen Modellierung von inno- 


\section{Substanzwertableitung...}

\begin{tabular}{lll}
\hline $\begin{array}{l}\text { k. Eingabe der Projekt- } \\
\text { konfiguration /-prämissen im } \\
\text { crossfunktionalen Team }\end{array}$ & $\begin{array}{l}\text {.. Parametereinstellungen ergeben } \\
\text { eine Konzeptbewertung für Kapazität } \\
\text { und Versuchsteile-/Werkzeugkosten }\end{array}$ & $\begin{array}{l}\text {... Einarbeitung des } \\
\text { wettbewerbsorientierten } \\
\text { Verbesserungsanspruchs von } \\
5 \% \text { p. a. für zukünftige Projekte }\end{array}$ \\
\hline
\end{tabular}

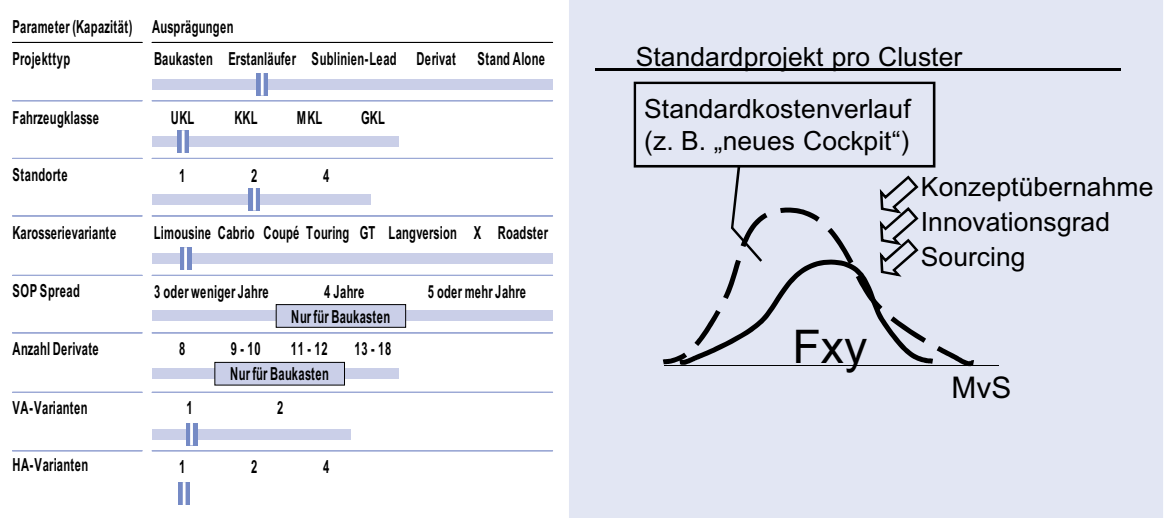

Output

- Crossfunktional vereinbarte Parameterausprägungen
Standardprojekt pro Cluster (z. B. "neues Cockpit“)
Standardkostenverlauf

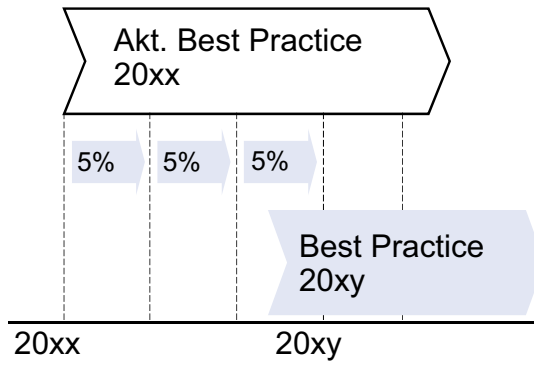

- Substanzwert

- Effizienzanspruch in Abhängigkeit SOP Ziel-Fzg.

Abb. 6: Der Substanzwert basiert auf interner Best Practice inkl. der vereinbarten Effizienzsteigerung

vativen Konzepten. Diese Umfänge müssen daher bei der Bewertung über Expertenschätzungen ergänzt werden. Das Führungssystem kann auch nicht die spätere detaillierte Ausplanung und finanzielle Bewertung eines Projektes ersetzen, die die Basis der konkreten Budgetierung und Zielführung auf Abteilungsebene darstellt.

Der Effizienzanspruch von $5 \%$ p. a. für den Entwicklungsprozess erscheint hoch, konnte aber in den vergangenen Jahren erreicht werden. Neue Ansätze zu weiter gespreizten Fahrzeugarchitekturen, übergreifenden Baukästen und noch leistungsfähigeren Systemen für Design, Konstruktion, Berechnung und (virtuelle) Absicherung lassen auch für die Zukunft deutliche Effizienzpotenziale erwarten. Eine regelmäßige Kalibrierung des Systems an Ist-Daten i. S. der Selbstschärfung stellt sicher, dass die aktuelle Best Practice abgebildet ist und unterschiedliche Effizienzfortschritte in den einzelnen Bereichen transparent gemacht werden, um so die Zielansprüche realistisch und wettbewerbsorientiert auszurichten.

Ebenso wichtig wie die jährliche Kalibrierung ist eine Überarbeitung des gesamten
Systems etwa alle drei bis fünf Jahre. Dabei müssen die Effekte modelliert und eingearbeitet werden, die sich zum Beispiel ergeben aus: neuen Entwicklungsprozessen (z. B. Internationalisierung oder stärkere Virtualisierung), angepassten Modulzuschnitten (z. B. erweiterte Baukastennutzung), der Verwendung neuer Materialien (z. B. Kohlefasereinsatz als Leichtbaumaßnahme) oder den Auswirkungen neuer Kunden- oder Gesetzesanforderungen (leistungsfähigere Bordnetze und Sensorik für Fahrerassistenzsysteme, spezifische Umfänge von Hybrid- und Elektrofahrzeugen oder neue Anforderungen an die Fahrzeugsicherheit). Dies führt teilweise zu zusätzlichen Modulen oder neuen Parametern und angepassten Kostensätzen bei bestehenden Modulen. Wesentlich für alle Kalibrierungen und Weiterentwicklungen sind vor allem eine hohe Buchungsqualität der Ist-Aufwendungen sowie die übersichtliche Dokumentation der Projektprämissen. Qualität und Nachvollziehbarkeit der Buchungen und Prämissen waren eine der großen Herausforderungen in der Modellierung, vor allem bei etwas weiter in der Vergangenheit liegenden Projekten. Hier konnten oft nur er-

fahrene Mitarbeiter mit „historischem Wissen" für die nötige Richtigstellung der Systemdaten sorgen.

Zusammenfassend lässt sich festhalten, dass der vorgestellte Ansatz sich in der Praxis bestens bewährt hat und durch seine logische und klare Struktur wesentlich zur Versachlichung der Diskussion über ,angemessene“ Entwicklungskosten für spezifische Projekte beigetragen hat.

\section{Keywords \\ - Automotive industry \\ - Lever for development efficiency \\ - Product management \\ - R\&D costs \\ - Standard cost curve \\ Summary}

To better manage R\&D costs, an approach is described that supports the validation and ranking of available technical options in an early stage of the process. Focus is given to the systematic determination of the relevant cost drivers, as obtained from previously completed projects. When paired with an agreed-upon annual efficiency gain, this method delivers an up-front comparison of required in- 
vestment totals once correlated with market potentials and cost specific technical options.

\section{Literatur}

Adelberger, W./Haft-Zboril, N,. Portfoliomanagement als Aufgabe der Optimierung von Rendite, Marktanteil und Ressourceneinsatz, in: Controlling, 25. Jg. (2013), H. 1, S. 41-48.

Bullinger, H.-J./Kiss-Preußinger, E./Spath, D., Automobilentwicklung in Deutschland, Stuttgart 2003.

Burghardt, M., Projektmanagement, 7. Aufl., Erlangen 2006.

Ehrlenspiel, K., Integrierte Produktentwicklung, 4. Aufl., München 2009.

Nagarajah, A., Methodisches Klären und Präzisieren der Aufgabenstellung, in: Feldhusen, J./Grote, K.-H., Pahl, G., Beitz, W., Konstruktionslehre, 4. Aufl., Wiesbaden 2013.

Hab, G./Wagner, R. Projektmanagement in der Automobilindustrie, Wiesbaden 2013.

Schlechtweg, A., Entscheidungsmodell für ein zeitorientiertes Entwicklungskosten-Controlling, Lohmar 2009.

Schorb, M., Verhaltensorientiertes F\&E-Controlling, München 1994.

Literaturtipps aus dem Online-Archiv der ConTROLLING:

- Timo Riedrich und Alexander Sasse, Ganzheitliche Planung und Steuerung von Innovationsprojekten - Verbindung von Target Costing und Earned Value Methode, Ausgabe 3/2005, S. 173-179.

- Guntram Herda und Peter Lorson, Controlling der Entwicklungsbudgets in der deutschen Automobilzulieferindustrie - Ergebnisse einer empirischen Studie, Ausgabe 7/2010, S. 399-404.

- Annehild Bramann, Controllingwirkungen aus der Bilanzierung von Forschungs- und Entwicklungsausgaben (FuE-Ausgaben) nach IAS 38, Ausgabe 2/2009, S. 97-105.

\section{Alle BWL-Begriffe - präzise und verständlich erklärt}

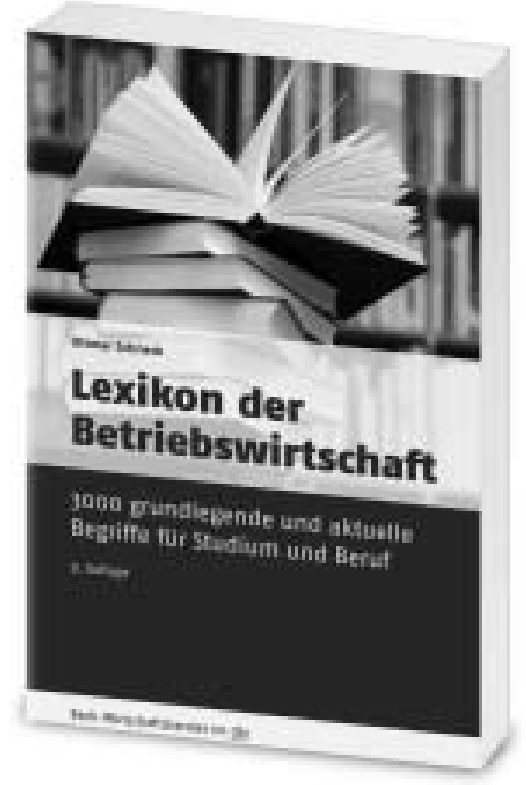

Herausgegeben von Prof. Dr. Ottmar Schneck. Mit Beiträgen von Prof. Dr. Ottmar Schneck, Prof. Dr. Klaus Hahn, Prof. Dr. Uwe Schramm und Dr. Matthias Stelzer. 9. Auflage. 2015. XIII, 1042 Seiten. Kartoniert $€ 19,90$ (dtv-Band 50942)

\section{Beantwortet alle BWL-Fragen}

Was ist Strategische Planung, welche Steueränderungen gibt es, was versteht man unter Break-Even-Analyse, Cashflow, Prozesskostenrechnung oder Balanced Scorecard und wo liegen die Besonderheiten der Konzernrechnungslegung?

\section{Mehr als 3000 Stichwörter}

und rund 200 Abbildungen erklären kompetent, präzise und verständlich das Wichtigste aus • Personal- und Unternehmensführung • Investition und Finanzierung • Marketing • Produktion - Beschaffung und Logistik • Kostenrechnung und Controlling - Rechnungslegung und Wirtschaftsprüfung • Steuern • Informationsmanagement.

\section{Beck-Wirtschaftsberater im dtv}

